\title{
Generalizations of Yang-Mills theory with nonlinear constitutive equations
}

\author{
Gerald A Goldin $^{1}$ and Vladimir M Shtelen ${ }^{2}$ \\ ${ }^{1}$ Departments of Mathematics and Physics, Rutgers University, Busch Campus, Piscataway, \\ NJ 08854, USA \\ ${ }^{2}$ Department of Mathematics, Rutgers University, Busch Campus, Piscataway, NJ 08854, USA \\ E-mail: gagoldin@dimacs.rutgers.edu and shtelen@math.rutgers.edu
}

Received 14 April 2004

Published 20 October 2004

Online at stacks.iop.org/JPhysA/37/10711

doi:10.1088/0305-4470/37/44/018

\begin{abstract}
We generalize classical Yang-Mills theory by extending nonlinear constitutive equations for Maxwell fields to non-Abelian gauge groups. Such theories may or may not be Lagrangian. We obtain conditions on the constitutive equations specifying the Lagrangian case, of which recently discussed non-Abelian BornInfeld theories are particular examples. Some models in our class possess nontrivial Galilean $(c \rightarrow \infty)$ limits; we determine when such limits exist and obtain them explicitly.
\end{abstract}

PACS numbers: 03.50.-z, 11.10.Lm, 11.10.Nx, 11.15.-q

\section{Introduction}

General equations for nonlinear, classical electromagnetic fields in media can be written beginning with Maxwell's equations for $\mathbf{E}, \mathbf{B}, \mathbf{D}$ and $\mathbf{H}$, and replacing the usual, linear constitutive equations by more general, nonlinear equations respecting Lorentz covariance. A general form for such systems was described by Fushchich, Shtelen and Serov; familiar special cases include Born-Infeld and Euler-Kockel electrodynamics [1,2]. In earlier work, we showed that certain nonlinear constitutive equations have well-defined Galilean-covariant limits as the speed of light $c \rightarrow \infty$, so that all four of Maxwell's equations remain valid [3]. This is in sharp contrast to linear electrodynamics, where Maxwell's equations are well-known to be incompatible with Galilean relativity [4, 5]. Since classical Yang-Mills theory can be understood as an extension of classical electromagnetism to non-Abelian gauge potentials, it is natural to similarly extend Maxwell fields with nonlinear constitutive equations, and to ask whether such extensions may also have Galilean-covariant limits when $c \rightarrow \infty$.

Non-Abelian generalizations of the Born-Infeld Lagrangian [6] (an excellent review of classical Born-Infeld theory is in [7]) have been known for some time, and recently have attracted renewed interest [8-12]. In this paper, we take a different approach, deriving 
generalizations of classical Yang-Mills theory as non-Abelian extensions of Maxwell systems together with Lorentz-covariant, in general nonlinear constitutive equations. Standard YangMills theory is a special case in this class of theories, with linear constitutive equations. Particular nonlinear constitutive equations correspond to the non-Abelian Born-Infeld theories. Our approach has the important advantage of being general enough to include Lagrangian and non-Lagrangian theories. In addition, since we directly generalize nonlinear Maxwell systems, we have the possibility of obtaining nontrivial Galilean-covariant (nonrelativistic) limits as $c \rightarrow \infty$.

In section 2 we review Maxwell's equations for media, and characterize the family of nonlinear constitutive equations that result in theories obtained from invariant Lagrangians. In section 3 we generalize appropriately from $U(1)$ to non-Abelian gauge theory. In section 4 we consider the nonrelativistic $c \rightarrow \infty$ limit. Then we show that with necessary modifications, certain Born-Infeld (Abelian or non-Abelian) Lagrangian functions lead to nontrivial theories having such a limit. We state our conclusions in section 5 .

\section{Nonlinear electrodynamics}

Here we use SI units, so that $c$ does not enter the definition of $\mathbf{E}$ or $\mathbf{B}$. We begin with the usual metric tensor $g^{\mu \nu}=\operatorname{diag}(1,-1,-1,-1), x^{\mu}=(c t, \mathbf{x}), x_{\mu}=g_{\mu \nu} x^{\nu}=(c t,-\mathbf{x})$ and $x_{\mu} x^{\mu}=c^{2} t^{2}-\mathbf{x}^{2}$. We have $\partial_{\mu} \equiv \partial / \partial x^{\mu}=[(1 / c) \partial / \partial t, \nabla]$, and we use the antisymmetric Levi-Civita tensor $\varepsilon^{\alpha \beta \mu \nu}$ with $\varepsilon^{0123}=1$.

The tensor fields constructed from vectors $\mathbf{E}, \mathbf{B}, \mathbf{D}$ and $\mathbf{H}$ are

$F_{\alpha \beta}=\left(\begin{array}{cccc}0 & \frac{1}{c} E_{1} & \frac{1}{c} E_{2} & \frac{1}{c} E_{3} \\ -\frac{1}{c} E_{1} & 0 & -B_{3} & B_{2} \\ -\frac{1}{c} E_{2} & B_{3} & 0 & -B_{1} \\ -\frac{1}{c} E_{3} & -B_{2} & B_{1} & 0\end{array}\right), \quad \quad F^{\alpha \beta}=g^{\alpha \mu} g^{\beta v} F_{\mu \nu}$

$\mathcal{F}^{\alpha \beta}=\frac{1}{2} \varepsilon^{\alpha \beta \mu \nu} F_{\mu \nu}=\left(\begin{array}{cccc}0 & -B_{1} & -B_{2} & -B_{3} \\ B_{1} & 0 & \frac{1}{c} E_{3} & -\frac{1}{c} E_{2} \\ B_{2} & -\frac{1}{c} E_{3} & 0 & \frac{1}{c} E_{1} \\ B_{3} & \frac{1}{c} E_{2} & -\frac{1}{c} E_{1} & 0\end{array}\right), \quad \mathcal{F}_{\alpha \beta}=g_{\alpha \mu} g_{\beta \nu} \mathcal{F}^{\mu \nu}$,

$G^{\alpha \beta}=\left(\begin{array}{cccc}0 & -c D_{1} & -c D_{2} & -c D_{3} \\ c D_{1} & 0 & -H_{3} & H_{2} \\ c D_{2} & H_{3} & 0 & -H_{1} \\ c D_{3} & -H_{2} & H_{1} & 0\end{array}\right), \quad G_{\alpha \beta}=g_{\alpha \mu} g_{\beta \nu} G^{\mu \nu}$

Maxwell's equations for media in SI units take the form [2]

$\nabla \times \mathbf{E}=-\frac{\partial \mathbf{B}}{\partial t}, \quad \nabla \cdot \mathbf{B}=0, \quad \nabla \times \mathbf{H}=\frac{\partial \mathbf{D}}{\partial t}+\mathbf{j}, \quad \nabla \cdot \mathbf{D}=\rho$, 
or in covariant form

$$
\partial_{\alpha} \mathcal{F}^{\alpha \beta}=0 ; \quad \partial_{\alpha} G^{\alpha \beta}=j^{\beta}, \quad j^{\beta}=(c \rho, \mathbf{j}) .
$$

The first equation in (2.4) allows us to introduce $A_{\mu}=(\phi,-\mathbf{A})$ so that

$$
\mathcal{F}^{\alpha \beta}=\epsilon^{\alpha \beta \mu \nu} \partial_{\mu} A_{\nu}, \quad F_{\mu \nu}=\partial_{\mu} A_{\nu}-\partial_{\nu} A_{\mu} .
$$

This system is invariant under the Lorentz group as well as the Galilei group; the choice between these symmetries rests in the constitutive equations [1, 3, 4]. The Lorentz invariant constitutive equations are

$$
\mathbf{D}=M \mathbf{B}+\frac{1}{c^{2}} N \mathbf{E}, \quad \mathbf{H}=N \mathbf{B}-M \mathbf{E},
$$

or in covariant form

$$
G^{\mu \nu}=N F^{\mu \nu}+c M \mathcal{F}^{\mu \nu} \equiv M_{1} \frac{\partial I_{1}}{\partial F_{\mu \nu}}+M_{2} \frac{\partial I_{2}}{\partial F_{\mu \nu}},
$$

where $M$ and $N$, or equivalently $M_{1}$ and $M_{2}$, are functions of the two Lorentz invariants

$$
I_{1}=\mathbf{B}^{2}-\frac{1}{c^{2}} \mathbf{E}^{2}=\frac{1}{2} F_{\mu \nu} F^{\mu \nu}, \quad I_{2}=\mathbf{B} \cdot \mathbf{E}=-\frac{c}{4} F_{\mu \nu} \mathcal{F}^{\mu \nu}
$$

The standard Maxwell equations for the vacuum correspond to $M=0, N=$ constant $=\left(\mu_{0}\right)^{-1}$, with $c^{2}=\left(\mu_{0} \epsilon_{0}\right)^{-1}$. A general form of an invariant Lagrangian for a nonlinear theory given by equations (2.4) and (2.7) may be written $\mathcal{L}=\mathcal{L}\left(I_{1}, I_{2}\right)$, where $I_{1}, I_{2}$ are given by (2.8). In such a theory, the tensor $G^{\mu \nu}$ becomes

$$
G^{\mu \nu}=-\frac{\partial \mathcal{L}}{\partial F_{\mu \nu}}=-\left(\frac{\partial \mathcal{L}}{\partial I_{1}}\right) 2 F^{\mu \nu}+c\left(\frac{\partial \mathcal{L}}{\partial I_{2}}\right) \mathcal{F}^{\mu \nu}
$$

In the above, following e.g. [7], the derivatives in (2.7) are evaluated by first imposing in equations (2.8) the constraints $F_{\mu \nu}=-F_{\nu \mu}, F^{\alpha \beta}=g^{\alpha \mu} g^{\beta \nu} F_{\mu \nu}$ and $\mathcal{F}^{\alpha \beta}=\frac{1}{2} \varepsilon^{\alpha \beta \mu \nu} F_{\mu \nu}$, and then taking the partial derivatives of $I_{1}$ and $I_{2}$; thus

$$
\frac{\partial I_{1}}{\partial F_{\mu \nu}}=2 F^{\mu \nu}, \quad \frac{\partial I_{2}}{\partial F_{\mu \nu}}=-c \mathcal{F}^{\mu \nu} .
$$

Comparison of equation (2.9) with (2.7) yields the conditions

$$
-2 \frac{\partial \mathcal{L}}{\partial I_{1}}=N, \quad \frac{\partial \mathcal{L}}{\partial I_{2}}=M,
$$

from which the compatibility condition for a Lagrangian theory reads

$$
2 \frac{\partial M}{\partial I_{1}}+\frac{\partial N}{\partial I_{2}}=0
$$

The well-known Born-Infeld Lagrangian is usually written as

$$
\mathcal{L}_{B I}=\frac{b^{2}}{\mu_{0} c^{2}}(1-R), \quad R=\sqrt{1+\frac{c^{2}}{b^{2}} I_{1}-\frac{c^{2}}{b^{4}} I_{2}^{2}},
$$

where $b$ is a maximum electric field strength (in the absence of magnetic field). If $b^{2}$ is very much larger than $\mathbf{E}^{2}$ and $c^{2} \mathbf{B}^{2}$, then $\mathcal{L}_{B I} \approx-\left(1 / 2 \mu_{0}\right) I_{1}$ and we recover linear Maxwell theory. But anticipating the discussion in section 4 , we remark here that in the limit as $c \rightarrow \infty, \mathcal{L}_{B I}$ tends to zero, while $c \mathcal{L}_{B I}$ approaches a well-defined, non-zero limit. 
Another example is Euler-Kockel electrodynamics [2]. Here, in the first approximation, one has $M=7 \lambda\left(\mu_{0}\right)^{-1} I_{2}$ and $N=\left(\mu_{0}\right)^{-1}\left(1-2 \lambda I_{1}\right)$, where $\lambda$ is a small parameter. The corresponding Lagrangian takes the form

$$
\mathcal{L}=-\frac{1}{2 \mu_{0}} I_{1}+2 \lambda I_{1}^{2}+\frac{7 \lambda}{2 \mu_{0}} I_{2}^{2},
$$

which, we remark, coincides with the 'toy model' generalization of the Maxwell Lagrangian discussed by DeLorenci et al [13].

\section{Generalization of Yang-Mills theory}

To generalize the nonlinear electrodynamics described in section 2 to non-Abelian gauge theory, we replace as usual the partial derivative $\partial_{\mu}$ by the commutator with the covariant derivative $D_{\mu}$, i.e. $\partial_{\mu} \rightarrow\left[D_{\mu}, \cdot\right]$, where

$$
D_{\mu}=\partial_{\mu}+\mathrm{i} g T^{\ell} W_{\mu}^{\ell},
$$

$g$ is the YM coupling constant, $T^{\ell}$ are the $N^{2}-1$ generators of $S U(N)$ and summation over $\ell$ is assumed. Then

$$
\left[D_{\mu}, D_{\nu}\right]=\mathrm{i} g F_{\mu \nu}, \quad F_{\mu \nu}=T^{\ell} F_{\mu \nu}^{\ell} .
$$

The field equations of the non-Abelian theory generalizing the nonlinear Maxwell equations (2.4) and (2.7) take the form

$$
\left[D_{\mu}, \mathcal{F}^{\mu \nu}\right]=0, \quad\left[D_{\mu}, G^{\mu \nu}\right]=J^{v},
$$

where $J^{v}$ is an external current, and the constitutive equations are to be written in a new way. Letting $u_{s}(s=1,2,3, \ldots, m)$ be a set of independent invariant functions of the Yang-Mills fields, we write

$$
G^{\ell \mu \nu}=\sum_{s=1}^{m} M_{s}\left(u_{1}, u_{2}, \ldots, u_{m}\right) \frac{\partial u_{s}}{\partial F_{\mu \nu}^{\ell}},
$$

where $M_{s}$ are functions of the invariants. For the gauge group $S U(N)$ we have no fewer than $m=5 N^{2}-11$ independent invariants, using the following simple argument of Roskies: since the gauge group $S U(N)$ has $N^{2}-1$ parameters, and the Lorentz group has six parameters, the number of components of $F_{\mu \nu}^{\ell}$ is $6\left(N^{2}-1\right)$. One can choose a Lorentz frame and an $\mathrm{O}(N)$ frame in which $6+\left(N^{2}-1\right)=5+N^{2}$ components vanish. There will then be $6\left(N^{2}-1\right)-\left(5+N^{2}\right)=5 N^{2}-11$ remaining components. Any invariant could be evaluated in this special frame, and therefore could be a function of these $5 N^{2}-11$ components. In particular, there are nine independent invariants for $S U(2)[14]$ :

$$
\begin{aligned}
& u_{1}=\operatorname{tr}(K), \quad u_{2}=-\frac{1}{2} \operatorname{tr}(J), \quad u_{3}=\frac{1}{4} \operatorname{tr}\left(J^{2}\right), \\
& u_{4}=-\operatorname{det}(J), \quad u_{5}=\operatorname{tr}\left(K^{2}\right), \quad u_{6}=\operatorname{det}(K), \\
& u_{7}=\operatorname{tr}(J K), \quad u_{8}=\frac{1}{6} \varepsilon_{i j k} F_{\mu}^{i v} F_{\nu}^{j \rho} F_{\rho}^{k \mu}, \quad u_{9}=-\frac{1}{6} c \varepsilon_{i j k} \mathcal{F}_{\mu}^{i \nu} \mathcal{F}_{\nu}^{j \rho} \mathcal{F}_{\rho}^{k \mu}
\end{aligned}
$$

where

$$
\begin{aligned}
& K_{i j}=\frac{1}{2} F_{\mu \nu}^{i} F^{j \mu \nu}=\mathbf{B}^{i} \cdot \mathbf{B}^{j}-\frac{1}{c^{2}} \mathbf{E}^{i} \cdot \mathbf{E}^{j}, \\
& J_{i j}=\frac{c}{2} F_{\mu \nu}^{i} \mathcal{F}^{j \mu \nu}=-\left[\mathbf{B}^{i} \cdot \mathbf{E}^{j}+\mathbf{B}^{j} \cdot \mathbf{E}^{i}\right],
\end{aligned}
$$


with $i, j, k=1,2,3$ being the $S U(2)$ algebra indices. The factors of $c$ in equations (3.5) and (3.6) have been introduced so that in all cases, the limit $c \rightarrow \infty$ results in survival of the leading terms.

With a little help from Maple, we calculated the explicit form of the invariants $u_{1}, \ldots, u_{9}$ in equations (3.5). In the notation that follows, the $\mathbf{B}^{\ell}$ (gauge components $\ell=1,2,3$ ) are vectors with spatial components $B_{1}^{\ell}, B_{2}^{\ell}$ and $B_{3}^{\ell}$. The Lorentz YM gauge invariants are as follows:

$$
\begin{aligned}
& u_{1}=\sum_{\ell=1}^{3}\left(\mathbf{B}^{\ell} \cdot \mathbf{B}^{\ell}-\frac{1}{c^{2}} \mathbf{E}^{\ell} \cdot \mathbf{E}^{\ell}\right) \\
& u_{2}=\sum_{\ell=1}^{3} \mathbf{B}^{\ell} \cdot \mathbf{E}^{\ell} \\
& u_{3}=\left(\mathbf{B}^{1} \cdot \mathbf{E}^{1}\right)^{2}+\left(\mathbf{B}^{2} \cdot \mathbf{E}^{2}\right)^{2}+\left(\mathbf{B}^{3} \cdot \mathbf{E}^{3}\right)^{2} \\
& +\frac{1}{2}\left[\left(\mathbf{B}^{1} \cdot \mathbf{E}^{2}+\mathbf{B}^{2} \cdot \mathbf{E}^{1}\right)^{2}+\left(\mathbf{B}^{2} \cdot \mathbf{E}^{3}+\mathbf{B}^{3} \cdot \mathbf{E}^{2}\right)^{2}+\left(\mathbf{B}^{3} \cdot \mathbf{E}^{1}+\mathbf{B}^{1} \cdot \mathbf{E}^{3}\right)^{2}\right], \\
& u_{4}=\operatorname{det}(J), \quad J_{i j}=-\left[\mathbf{B}^{i} \cdot \mathbf{E}^{j}+\mathbf{B}^{j} \cdot \mathbf{E}^{i}\right], \\
& u_{5}=\left(\mathbf{B}^{1} \cdot \mathbf{B}^{1}-\frac{1}{c^{2}} \mathbf{E}^{1} \cdot \mathbf{E}^{1}\right)^{2}+\left(\mathbf{B}^{2} \cdot \mathbf{B}^{2}-\frac{1}{c^{2}} \mathbf{E}^{2} \cdot \mathbf{E}^{2}\right)^{2}+\left(\mathbf{B}^{3} \cdot \mathbf{B}^{3}-\frac{1}{c^{2}} \mathbf{E}^{3} \cdot \mathbf{E}^{3}\right)^{2} \\
& +2\left[\left(\mathbf{B}^{1} \cdot \mathbf{B}^{2}-\frac{1}{c^{2}} \mathbf{E}^{1} \cdot \mathbf{E}^{2}\right)^{2}+\left(\mathbf{B}^{1} \cdot \mathbf{B}^{3}-\frac{1}{c^{2}} \mathbf{E}^{1} \cdot \mathbf{E}^{3}\right)^{2}+\left(\mathbf{B}^{2} \cdot \mathbf{B}^{3}-\frac{1}{c^{2}} \mathbf{E}^{2} \cdot \mathbf{E}^{3}\right)^{2}\right], \\
& u_{6}=\operatorname{det}(K), \quad K_{i j}=\mathbf{B}^{i} \cdot \mathbf{B}^{j}-\frac{1}{c^{2}} \mathbf{E}^{i} \cdot \mathbf{E}^{j}, \\
& u_{7}=\left(\mathbf{B}^{1} \cdot \mathbf{B}^{2}-\frac{1}{c^{2}} \mathbf{E}^{1} \cdot \mathbf{E}^{2}\right)\left(\mathbf{E}^{1} \cdot \mathbf{B}^{2}+\mathbf{B}^{1} \cdot \mathbf{E}^{2}\right)+\left(\mathbf{B}^{1} \cdot \mathbf{B}^{3}-\frac{1}{c^{2}} \mathbf{E}^{1} \cdot \mathbf{E}^{3}\right) \\
& \times\left(\mathbf{E}^{1} \cdot \mathbf{B}^{3}+\mathbf{B}^{1} \cdot \mathbf{E}^{3}\right)+\left(\mathbf{B}^{2} \cdot \mathbf{B}^{3}-\frac{1}{c^{2}} \mathbf{E}^{2} \cdot \mathbf{E}^{3}\right)\left(\mathbf{E}^{2} \cdot \mathbf{B}^{3}+\mathbf{B}^{2} \cdot \mathbf{E}^{3}\right), \\
& u_{8}=\left(\mathbf{B}^{1} \times \mathbf{B}^{2}\right) \cdot \mathbf{B}^{3}-\frac{1}{c^{2}}\left[\left(\mathbf{E}^{1} \times \mathbf{E}^{2}\right) \cdot \mathbf{B}^{3}+\left(\mathbf{E}^{2} \times \mathbf{E}^{3}\right) \cdot \mathbf{B}^{1}+\left(\mathbf{E}^{3} \times \mathbf{E}^{1}\right) \cdot \mathbf{B}^{2}\right], \\
& u_{9}=-\frac{1}{2} \epsilon_{i j k}\left(\mathbf{B}^{i} \times \mathbf{B}^{j}\right) \cdot \mathbf{E}^{k}+\frac{1}{c^{2}}\left(\mathbf{E}^{1} \times \mathbf{E}^{2}\right) \cdot \mathbf{E}^{3} .
\end{aligned}
$$

Note that $u_{2}, u_{3}$ and $u_{4}$ are independent of $c$ and, therefore, they will be the same in the Galilean limit $(c \rightarrow \infty)$.

There are now Lagrangian and non-Lagrangian theories determined by equations (3.3) and (3.4). In a Lagrangian theory, the constitutive equations are

$$
G^{\ell \mu \nu}=-\frac{\partial \mathcal{L}}{\partial F_{\mu \nu}^{\ell}}=-\sum_{s=1}^{m} \frac{\partial \mathcal{L}}{\partial u_{s}} \frac{\partial u_{s}}{\partial F_{\mu \nu}^{\ell}} .
$$

Thus equations (3.3) and (3.4) determine a Lagrangian theory if and only if the coefficients $M_{s}$ in (3.4) can be written as $M_{s}=-\partial \mathcal{L} / \partial u_{s}$ for some scalar-valued function $\mathcal{L}=\mathcal{L}\left(u_{1}, u_{2}, \ldots, u_{m}\right)$. The corresponding restrictions on the $M_{s}$ are the compatibility conditions resulting from the equalities of the mixed derivatives of $\mathcal{L}$ with respect to $u_{r}$ and $u_{s}$, i.e., $\partial M_{s} / \partial u_{r}=\partial M_{r} / \partial u_{s}$ $(\forall r, s=1,2, \ldots, m)$.

In particular, one obtains non-Abelian versions of Born-Infeld or Euler-Kockel theory by taking various generalizations of the respective Lagrangians discussed in section 2. For example, a Born-Infeld Lagrangian proposed in [8] for non-Abelian chromodynamics (CD) 
is given by

$\mathcal{L}_{B I C D}=\frac{b^{2}}{\mu_{0} c^{2}}\left(1-R_{C D}\right), \quad R_{C D}=\sqrt{1+\frac{c^{2}}{b^{2}} u_{1}-\frac{c^{2}}{3 b^{4}}\left(u_{2}^{2}+2 u_{3}\right)}$.

In the Abelian case, $u_{1}$ is $I_{1}, u_{2}$ is $I_{2}$ and $u_{3}$ reduces to $u_{2}^{2}=I_{2}^{2}$; so that equation (3.9) becomes the same as equation (2.12).

\section{A framework for non-Abelian Galilean theories}

Let us consider the nonrelativistic limit of the equations derived in section 2. Galilean symmetry transformations (the $c \rightarrow \infty$ limit of Lorentz transformations) have the form

$$
t^{\prime}=t, \quad \mathbf{x}^{\prime}=\mathbf{x}-\mathbf{v} t, \quad\left(\mathbf{E}^{\ell}\right)^{\prime}=\mathbf{E}^{\ell}+\mathbf{v} \times \mathbf{B}^{\ell}, \quad\left(\mathbf{B}^{\ell}\right)^{\prime}=\mathbf{B}^{\ell} .
$$

As is well known, there is no nonrelativistic limit of the standard Yang-Mills equations. This is because the linear constitutive equations $G^{\ell \mu \nu}=\left(1 / \mu_{0}\right) F^{\ell \mu \nu}$ break the Galilean symmetry. But our equations (3.3) and (3.4) can have a $c \rightarrow \infty$ limit, provided the constitutive equations are also nonlinear. One obtains such a Galilean non-Abelian gauge theory from equations (3.3) and (3.4) by writing these equations explicitly in terms of $\mathbf{E}^{\ell}, \mathbf{B}^{\ell}, \mathbf{D}^{\ell}$ and $\mathbf{H}^{\ell}$, and then taking the limit as $c \rightarrow \infty$. The equations of motion (3.3) will always be the same as in the relativistic theory, as the factors of $c$ cancel; only the constitutive equations (3.4) will be different.

The Galilean YM gauge invariants $\hat{u}_{1}, \ldots, \hat{u}_{9}$ are as follows

$$
\begin{aligned}
& \hat{u}_{1}=\sum_{\ell=1}^{3} \mathbf{B}^{\ell} \cdot \mathbf{B}^{\ell}, \\
& \hat{u}_{2}=u_{2}=\sum_{\ell=1}^{3} \mathbf{B}^{\ell} \cdot \mathbf{E}^{\ell}, \\
& \hat{u}_{3}=u_{3}=\left(\mathbf{B}^{1} \cdot \mathbf{E}^{1}\right)^{2}+\left(\mathbf{B}^{2} \cdot \mathbf{E}^{2}\right)^{2}+\left(\mathbf{B}^{3} \cdot \mathbf{E}^{3}\right)^{2} \\
& \quad+\frac{1}{2}\left[\left(\mathbf{B}^{1} \cdot \mathbf{E}^{2}+\mathbf{B}^{2} \cdot \mathbf{E}^{1}\right)^{2}+\left(\mathbf{B}^{2} \cdot \mathbf{E}^{3}+\mathbf{B}^{3} \cdot \mathbf{E}^{2}\right)^{2}+\left(\mathbf{B}^{3} \cdot \mathbf{E}^{1}+\mathbf{B}^{1} \cdot \mathbf{E}^{3}\right)^{2}\right], \\
& \hat{u}_{4}=u_{4}=\operatorname{det}\left(\mathbf{B}^{i} \cdot \mathbf{E}^{j}+\mathbf{B}^{j} \cdot \mathbf{E}^{i}\right), \\
& \hat{u}_{5}=\left(\mathbf{B}^{1} \cdot \mathbf{B}^{1}\right)^{2}+\left(\mathbf{B}^{2} \cdot \mathbf{B}^{2}\right)^{2}+\left(\mathbf{B}^{3} \cdot \mathbf{B}^{3}\right)^{2}+2\left[\left(\mathbf{B}^{1} \cdot \mathbf{B}^{2}\right)^{2}+\left(\mathbf{B}^{1} \cdot \mathbf{B}^{3}\right)^{2}+\left(\mathbf{B}^{2} \cdot \mathbf{B}^{3}\right)^{2}\right], \\
& \hat{u}_{6}=\operatorname{det}\left(\mathbf{B}^{i} \cdot \mathbf{B}^{j}\right), \\
& \hat{u}_{7}=\left(\mathbf{B}^{1} \cdot \mathbf{B}^{2}\right)\left(\mathbf{E}^{1} \cdot \mathbf{B}^{2}+\mathbf{B}^{1} \cdot \mathbf{E}^{2}\right)+\left(\mathbf{B}^{1} \cdot \mathbf{B}^{3}\right)\left(\mathbf{E}^{1} \cdot \mathbf{B}^{3}+\mathbf{B}^{1} \cdot \mathbf{E}^{3}\right)\left(\mathbf{B}^{2} \cdot \mathbf{B}^{3}\right)\left(\mathbf{E}^{2} \cdot \mathbf{B}^{3}+\mathbf{B}^{2} \cdot \mathbf{E}^{3}\right), \\
& \hat{u}_{8}=\frac{1}{6} \epsilon_{i j k}\left(\mathbf{B}^{i} \times \mathbf{B}^{j}\right) \cdot \mathbf{B}^{k}=\left(\mathbf{B}^{1} \times \mathbf{B}^{2}\right) \cdot \mathbf{B}^{3}, \\
& \hat{u}_{9}=-\frac{1}{2} \epsilon_{i j k}\left(\mathbf{B}^{i} \times \mathbf{B}^{j}\right) \cdot \mathbf{E}^{k} .
\end{aligned}
$$

Using (4.1) one can check directly (Maple helps) that, indeed, $\hat{u}_{1}, \ldots, \hat{u}_{9}$ are Galilean invariants.

Let us look at some Born-Infeld theories in the Galilean limit. In the Abelian case, we obtain constitutive equations of the form of equation (2.6), with

$$
M=\frac{I_{2}}{\mu_{0} b^{2} R}, \quad N=\frac{1}{\mu_{0} R} .
$$


In the limit as $c \rightarrow \infty$, we have $I_{1} \rightarrow \hat{I}_{1}=\mathbf{B}^{2}$ and $I_{2}=\hat{I}_{2}=\mathbf{B} \cdot \mathbf{E}$. But in this limit, $R \approx(c / b)\left[\hat{I}_{1}-\hat{I}_{2}^{2} / b^{2}\right]^{1 / 2}$, so that $M$ and $N$ do not approach well-defined nonzero limits. This suggests the introduction of a modification of the Born-Infeld Lagrangian. For example, one possibility is to replace $R$ in equation $(2.12)$ by

$$
\tilde{R}=\sqrt{1+\frac{c^{2}}{b^{2}}\left[\left(1+\lambda_{1} c^{2}\right) I_{1}-\frac{1}{b^{2}}\left(1+\lambda_{2} c^{2}\right) I_{2}^{2}\right]},
$$

where $\lambda_{1}, \lambda_{2}$ have the dimensionality of inverse velocity squared. Then in the limit when $c \rightarrow \infty$, we obtain the Galilean constitutive equations

$$
\mathbf{D}=\hat{M} \mathbf{B}, \quad \mathbf{H}=\hat{N} \mathbf{B}-\hat{M} \mathbf{E},
$$

where

$$
\hat{M}=\frac{\lambda_{2} \hat{I}_{2}}{\mu_{0} b \sqrt{\lambda_{1} \hat{I}_{1}-\lambda_{2} \hat{I}_{2}^{2} / b^{2}}}, \quad \hat{N}=\frac{b \lambda_{1}}{\mu_{0} \sqrt{\lambda_{1} \hat{I}_{1}-\lambda_{2} \hat{I}_{2}^{2} / b^{2}}}
$$

Similarly, in the non-Abelian case, we obtain a well-defined Galilean limit for the YangMills constitutive equations (3.8) if we modify $R_{C D}$ in equation (3.9) to be

$$
\tilde{R}_{C D}=\sqrt{1+\frac{c^{2}}{b^{2}}\left(1+\lambda_{1} c^{2}\right) u_{1}-\frac{c^{2}}{3 b^{4}}\left(1+\lambda_{2} c^{2}\right)\left(u_{2}^{2}+2 u_{3}\right)} .
$$

Then, with $c \rightarrow \infty$,

$$
\mathbf{D}^{\ell}=\frac{\lambda_{2}\left(\hat{u}_{2}+2 \mathbf{B}^{\ell} \cdot \mathbf{E}^{\ell}\right)}{3 \mu_{0} b \sqrt{\lambda_{1} \hat{u}_{1}-\frac{\lambda_{2}}{3 b^{2}}\left(\hat{u}_{2}^{2}+2 \hat{u}_{3}\right)}} \mathbf{B}^{\ell}
$$

and

$$
\mathbf{H}^{\ell}=\frac{b \lambda_{1}}{\mu_{0} \sqrt{\lambda_{1} \hat{u}_{1}-\frac{\lambda_{2}}{3 b^{2}}\left(\hat{u}_{2}^{2}+2 \hat{u}_{3}\right)}} \mathbf{B}^{\ell}-\frac{\lambda_{2}\left(\hat{u}_{2}+2 \mathbf{B}^{\ell} \cdot \mathbf{E}^{\ell}\right)}{3 \mu_{0} b \sqrt{\lambda_{1} \hat{u}_{1}-\frac{\lambda_{2}}{3 b^{2}}\left(\hat{u}_{2}^{2}+2 \hat{u}_{3}\right)}} \mathbf{E}^{\ell} .
$$

Note that in equations (4.8) and (4.9) there is no summation over $\ell$, while $\hat{u}_{1}, \hat{u}_{2}, \hat{u}_{3}$ are the Galilean invariants given by equations (4.2).

We close this section with the remark that the nonlinear gauge theory described here can be set up usefully with a 'Galilei friendly' metric tensor, respecting the fact that space and time require different units (independent of $c$ ) if the Galilean limit is to be meaningful. By setting $\hat{g}^{\mu \nu}=\operatorname{diag}\left(1 / c^{2},-1,-1,-1\right)$ and $\hat{g}_{\mu \nu}=\operatorname{diag}\left(c^{2},-1,-1,-1\right)$, so that $x^{\mu}=(t, \mathbf{x}), x_{\mu}=$ $\hat{g}_{\mu \nu} x^{\nu}=\left(c^{2} t,-\mathbf{x}\right)$ and $x_{\mu} x^{\mu}=c^{2} t^{2}-\mathbf{x}^{2}$, we obtain in place of equations (2.1)-(2.2) matrix expressions for $\hat{F}_{\alpha \beta}, \hat{\mathcal{F}}^{\alpha \beta}$ and $\hat{G}^{\alpha \beta}$ in terms of the fields $\mathbf{E}$ and $\mathbf{B}$ that involve no factors of $c$, while factors of $1 / c^{2}$ or $c^{2}$ occur in the expressions for the other field strengths. With such a choice, taking the limit $c \rightarrow \infty$ in the relativistic equations is straightforward. The equations of motion (3.3) do not involve $c$ and do not change, while the constitutive equations (3.4) change as $c \rightarrow \infty$.

\section{Conclusion}

We have seen how it is possible to generalize nonlinear Maxwell systems directly to the case of non-Abelian gauge groups, thus obtaining generalized Yang-Mills theories associated with 
nonlinear constitutive equations for the fields. Such a theory may or may not be derivable from a Lagrangian function. Our construction allows for either situation, and permits one to determine directly from the constitutive equations whether or not a Lagrangian formulation is possible.

In particular, our approach highlights the possibility of obtaining nontrivial Galileancovariant (nonrelativistic) limits of these theories as $c \rightarrow \infty$. We have seen that such limits exist in some cases, but not in all. We believe they have potential application in contexts where Galilean theories are coupled with nonlinear electromagnetic fields or their non-Abelian counterparts-for example, in nonlinear Schrödinger theory as described in [15] and discussed in [3], or in non-Abelian fluid mechanics [16-18]. They are also potentially applicable, as noted in [3], to electromagnetic fields in condensed matter where the nonlinearity is extremely strong, and as effective, low-energy limits in string theory.

\section{Acknowledgment}

GG thanks R Kerner for interesting discussions and helpful suggestions.

\section{References}

[1] Fushchich W I, Shtelen V M and Serov N I 1993 Symmetry Analysis and Exact Solutions of Equations of Nonlinear Mathematical Physics (Dordrecht: Kluwer)

[2] Jackson J D 1999 Classical Electrodynamics 3rd edn (New York: Wiley)

[3] Goldin G A and Shtelen V M 2001 Phys. Lett. A 279321

[4] Le Bellac M and Levy-Leblond J-M 1973 Nuovo Cimento B 14217

[5] Brown H R and Holland P R 1999 Am. J. Phys. 67204

[6] Born M and Infeld L 1934 Proc. R. Soc. 144425

[7] Bialynicki-Birula I 1983 Nonlinear electrodynamics: variations on a theme by Born and Infeld Quantum Theory of Particles and Fields ed B Jancewicz and J Lukierski (Singapore: World Scientific) pp 31-48

[8] Hagiwara T 1981 J. Phys. A: Math. Gen. 143059

[9] Tseytlin A A 1997 Nucl. Phys. B 50141

[10] Park J-H 1999 Phys. Lett. B 458471

[11] Gal'tsov D and Kerner R 2000 Phys. Rev. Lett. 845955

[12] Serié E, Masson T and Kerner R 2003 Preprint hep-th/030710 v2

[13] DeLorenci V A, Klippert R, Novello M and Salim J M 2002 Phys. Rev. D 65063501

[14] Roskies R 1977 Phys. Rev. D 141722

[15] Doebner H-D, Goldin G A and Nattermann P 1999 J. Math. Phys. 4049

[16] Jackiw R 2002 A Particle Theorist's Lectures on Supersymmetric Non-Abelian Fluid Mechanics and d-Branes (New York: Springer)

[17] Bistrovic B, Jackiw R, Li H, Nair V P and Pi S Y 2002 Preprint hep-th/0210143 v2

[18] Grundland A M and Hariton A J 2004 J. Math. Phys. 453239 